\title{
LOS ODS, EN LA ESTRATEGIA EMPRESARIAL. EL CASO SUEZ
}

\author{
Autora: Dulcinea Meijide Vidal \\ dulcinea.meijide@suez.com \\ Directora de Desarrollo Sostenible SUEZ España y responsable DIRSE Cataluña
}

\section{Resumen}

Los Objetivos de Desarrollo Sostenible (ODS), adoptados en la Asamblea de la ONU el 25 de septiembre del 2015, están guiando, desde su nacimiento, políticas, planes de acción y estrategias de gobiernos, instituciones, empresas, entidades del tercer sector y ciudadanía en general, con el fin de contribuir a mejorar el presente y el futuro de las personas y del planeta. A diferencia de sus predecesores, los Objetivos del Milenio, los ODS realizan una llamada a la acción explícita a todas las empresas, para que apliquen su creatividad e innovación para dar solución a los grandes retos del desarrollo sostenible.

Desde el mundo empresarial, cada vez son más las empresas que están integrando los ODS en sus estrategias empresariales, creando compromisos concretos y medibles que contribuyan al desarrollo sostenible.

En este documento, analizaremos la necesidad de contribuir a los ODS desde la empresa, los beneficios que los ODS pueden aportar a las organizaciones y cómo integrarlos en las estrategias empresariales, compartiendo el caso de SUEZ en España. 
Palabras clave: Objetivos Desarrollo Sostenible; Agenda 2030; estrategia empresarial en sostenibilidad; organizaciones responsables; retos globales.

\title{
SDG, In the business strategy. Suez case
}

\begin{abstract}
Sustainable Development Goals (SDG), adopted by United Nations on $25^{\text {th }}$ September 2015, are guiding politics, action plans and strategies of governments, institutions, business organizations, NGO's and citizens, with the aim of improving the present and the future of the people and the planet. In contrast to the Millennium Development Goals, SDG make a call for an action for businesses to apply its creativity and innovation in global and sustainable goals solutions.

In business, more and more business organizations are integrating SDG's into their core business strategies and making concrete and measurable commitments for the sustainable development.

In this document, we analyze the business necessity to contribute SDG's, its benefits and how to integrate SDG's into the core business strategy, focusing on SUEZ Spain best practice.
\end{abstract}

Keywords: Sustainable Development Goals; 2030 Agenda; business strategy on sustainability; responsive organizations, global challenges.

Fecha de recepción: 18/11/2019.

Fecha de aceptación: 20/11/2019.

\section{LOS ODS, UNA NUEVA HOJA DE RUTA MUNDIAL}

Los Objetivos de Desarrollo Sostenible (ODS) son la hoja de ruta global para el desarrollo sostenible, con un horizonte de 15 años, aprobada en la 70ํ Asamblea General de las Naciones Unidas celebrada en septiembre de 2015, tras la firma de 193 jefes de Estado y de Gobierno mundiales y un proceso consultivo mundial sin precedentes de más de tres años.

Los 17 ODS y sus 169 metas configuran ahora, cuatro años después, la agenda global (Agenda 2030) que marcará la acción global y local hasta 2030 para lograr un objetivo común: el desarrollo mundial sostenible. La Agenda 2030 afronta los problemas más acuciantes de nuestro planeta con el fin de mejorar el presente y el futuro de las personas y el planeta, asegurando el bienestar, la justicia y la 
prosperidad. Se vertebra mediante 17 objetivos de desarrollo sostenible, a través de los cuales se propone abordar los grandes retos globales, como la lucha contra la pobreza o el cambio climático hasta la educación, salud, la igualdad de género, la paz o las ciudades sostenibles. Cada ODS incluye diferentes metas (en total 169) que contribuyen al cumplimiento del objetivo.

Los ODS promueven la acción en 5 esferas de vital importancia, conocidas como las 5P: personas (People), planeta (Planet), prosperidad (Prosperity), paz (Peace) y alianzas (Partnership). Se trata de 17 compromisos ambiciosos, universales, medibles e interconectados en todas sus dimensiones y en todos los niveles. Y a diferencia de los anterior Objetivos de Desarrollo del Milenio, las empresas adquieren un papel clave y relevante, siendo consideradas agentes clave para lograr el desarrollo sostenible, tanto a nivel global como local. Los ODS hacen una llamada a todas las empresas para que apliquen su creatividad e innovación con el objetivo de ofrecer soluciones a los retos globales y locales del desarrollo sostenible. Así, pues, Ban Kimoon, secretario general de Naciones Unidas en 2015, señaló en la presentación de la Agenda 2030 en un encuentro de la Red Española de Pacto Mundial, que "en el nuevo escenario, las empresas tienen un papel fundamental que desempeñar en el éxito de cada una de las metas globales".

Otra de las claves de la Agenda 2030 son las alianzas, objeto central del último ODS. La colaboración entre los distintos sectores de la sociedad es decisiva para lograr el desarrollo sostenible. A través de la Agenda 2030, y teniendo en cuenta el alcance global y la ambición de los ODS, todos los sectores de la sociedad son reconocidos como actores clave del desarrollo sostenible. De este modo, el ODS 17 convierte las alianzas y la cooperación entre la sociedad civil, las empresas, las administraciones públicas y el tercer sector en una herramienta necesaria para alcanzar cada una de las metas de la Agenda 2030. En este sentido, según Ban Ki Moon, "para aplicar con éxito la Agenda 2030 para el Desarrollo Sostenible, debemos pasar rápidamente de los compromisos a la acción. Para ello, necesitamos alianzas sólidas, inclusivas e integradas a todos los niveles”.

\section{II. ¿POR QUÉ ES NECESARIO INTEGRAR LOS ODS EN LAS ORGANIZACIONES?}

Por primera vez, la ONU reconoció la fuerza transformadora de las empresas al ser la principal fuente mundial de la actividad económica y su liderazgo para mejorar el nivel de vida en todo el mundo. Por ello, las empresas deben jugar un papel fundamental en el cumplimiento de los desafíos planteados en la Agenda 2030. Además de su importancia en las agendas internacionales y nacionales, los ODS deben ser un must indispensable del discurso y la estrategia empresarial. 
Pero... ¿qué implicaciones se derivan de incorporar los ODS en la estrategia empresarial?

Los ODS ofrecen una oportunidad a las empresas de crear una narrativa clara y diferenciadora respecto a otras organizaciones dentro de un esquema complejo (17 objetivos y 169 metas). Y todo ello, con un lenguaje común, homogéneo, que permite mostrar y comparar la contribución de las empresas a los retos sociales y del planeta.

Así mismo, los ODS obligan a las empresas a realizar un autoexamen, identificando las áreas de impacto positivo o negativo, en relación con los retos concretos propuestos por esta hoja de ruta. Sólo de este modo, se puede realizar un plan de acción y evaluar y medir la contribución a la Agenda 2030.

Otra de las claves de la alineación empresarial con los ODS es la oportunidad de ejercer un liderazgo fuerte y visionario de los CEO de las empresas que ayude a lograr la transformación necesaria para dar paso al crecimiento económico inclusivo y sostenible.

Con la Agenda 2030, las organizaciones se ven obligas a entender mejor los riesgos emergentes en materia de operación, regulación y reputación.

Además de las implicaciones comentadas, los ODS ofrecen los siguientes beneficios para las organizaciones empresariales:

- Permiten identificar y desarrollar nuevas oportunidades de negocio vinculadas a su cumplimiento (nuevos productos o servicios o nuevas formas de actividad), con soluciones innovadoras y transformadoras.

- Fortalecen las relaciones y el diálogo con los grupos de relación: optimizan las relaciones con los clientes, mejoran las relaciones con los clientes, aumentan el engagement de los trabajadores y el atractivo de la empresa para la captura del talento...

- Potencian el valor del desarrollo sostenible empresarial y la responsabilidad social corporativa: los ODS pueden ayudar a reforzar la introducción de otro tipo de criterios, además de los económicos, en la toma de decisiones de cada una de las áreas de las empresas.

- Aumentan la estabilidad de las empresas, mercados y sociedades. Invertir para lograr los ODS facilita el crecimiento y la estabilidad de la sociedad, los mercados y las empresas y, por lo tanto, la perdurabilidad y la sostenibilidad de las organizaciones. La Agenda 2030, además, fomenta la transparencia y el buen gobierno en los mercados y empresas.

- Mejoran la comunicación corporativa usando un lenguaje común y un propósito compartido. Los ODS ofrecen un marco común a las empresas para su rendición de cuentas, su contribución y desempeño.

- Generan nuevas alianzas con nuevos actores, o con actores no considerados relevantes hasta ahora, con los que se comparten retos globales o locales. 
- Como conclusión, los ODS presentan oportunidades para las organizaciones potenciando el desarrollo sostenible, las alianzas, el liderazgo, las relaciones y la comunicación con los grupos de relación, y nuevas oportunidades de negocio que permitan hacer frente a los retos más grandes del mundo en materia de desarrollo sostenible. Las empresas líderes serán las que demuestren cómo sus negocios ayudan a avanzar en el desarrollo sostenible, minimizando los impactos negativos y maximizando los positivos en las personas y en el planeta.

\section{III. ¿CÓMOINTEGRARLOSODSENLAESTRATEGIA EMPRESARIAL? EL CASO DE SUEZ}

Tal y como se ha mencionado, es necesario alinear la estrategia de la empresa a los ODS. Para ello, analizaremos cómo integrar los ODS en la estrategia a través del caso de SUEZ en España.

Para llevar a cabo dicha integración, en SUEZ se han seguido las siguientes fases:

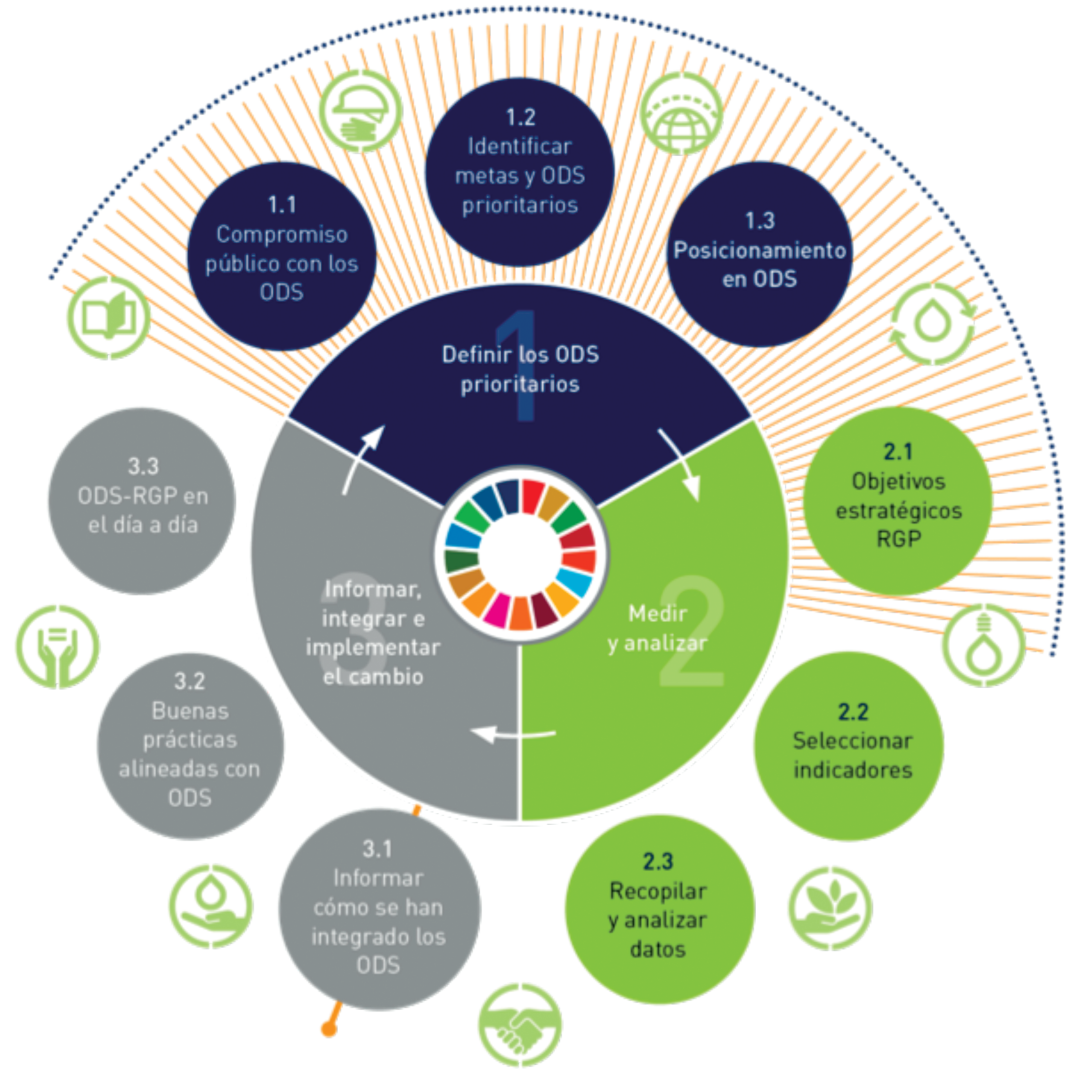

icade. Revista cuatrimestral de las Facultades de Derecho y Ciencias Económicas y Empresariales, nº 108 septiembre-diciembre 2019, ISSN: 2341-0841 


\section{DEFINIR LOS ODS PRIORITARIOS}

Para poder vincular los ODS con la actividad de una empresa y realizar un plan de acción, es necesario conocer en profundidad los 17 ODS y las 169 metas asociadas.

En SUEZ se llevó a cabo un exhaustivo escaneo de cada una de las metas identificando aquellas en las que, por su actividad, y teniendo en cuenta los impactos positivos y negativos de la actividad de SUEZ, estaban directamente relacionados con el negocio.

Posteriormente, se hizo público el compromiso de SUEZ en España con los ODS y se inició la tarea de priorizar los ODS más vinculados con su actividad. Contribuir a las 169 metas de la Agenda 2030 es un reto demasiado ambicioso. Por ello, es necesario priorizar los ODS a los que contribuir en función de varios factores como la actividad de la empresa, su propósito, su estrategia, los impactos positivos o negativos de su actividad en toda la cadena de valor, la materialidad, el mapa de los grupos de relación...

En SUEZ, se llevó a cabo un análisis de los 17 ODS y sus 169 metas con el fin de identificar los ODS prioritarios a los que contribuir. Garantizar el acceso al agua y al saneamiento (ODS 6), directamente relacionado con su actividad, el fin de la pobreza (ODS 1), vinculado a los esfuerzos en garantizar el acceso al agua a colectivos en situación de vulnerabilidad, y la lucha contra el cambio climático (ODS 13), que afecta a la disponibilidad del recurso agua, son los ODS prioritarios para SUEZ, junto con las alianzas (ODS 17).

A partir de este momento, con las principales metas y ODS identificados, se inició el proceso de definición del posicionamiento de SUEZ en relación a los ODS, coincidiendo, además, con el cambio de plan estratégico de desarrollo sostenible (2017-2021).

\section{ESTABLECER OBJETIVOS, MEDIR Y ANALIZAR}

Para poder establecer objetivos de sostenibilidad alineados con los ODS, es importante que éstos sean:

- Concisos: selección de ODS vinculados con la actividad

- Concretos: y asociados a metas específicas de los ODS

- Vinculados a la estrategia de la empresa

- Ambiciosos y claros

- Evaluables con indicadores (KPI) asociados

- Significativos, con un impacto directo sobre el negocio

Con motivo del cambio de plan estratégico de desarrollo sostenible, en SUEZ España se desarrolló durante todo el año 2016 el proyecto de definición del nuevo plan, bajo los siguientes criterios: 
a. Contando con la participación y el diálogo de todas las áreas de la organización en un proceso de co-creación, diseño, ejecución y seguimiento del Plan.

b. Teniendo en cuenta el análisis de materialidad, como punto de partida.

c. Con la participación de otros grupos de relación externos.

d. Y siempre, alineado con los ODS.

De este modo, se identificaron los objetivos estratégicos prioritarios basados en dos ejes: a. la mejora de la calidad de vida de las personas y b. la preservación del planeta, promoviendo un modelo de economía circular en la actividad de SUEZ. Y todo ello, teniendo en cuenta los ejes de innovación y alianzas, como motores de la sostenibilidad. A partir de este momento, y en colaboración con las áreas transversales y con otros expertos y grupos de relación externos, se fijaron 10 compromisos medibles y cuantificables, que se han convertido en la hoja de ruta de la empresa, impulsada por la dirección y por todas las áreas de la organización. Los 10 compromisos, vinculados cada uno a un ODS, conforman el REwater Global Plan (2017-2021).

Cada compromiso lleva su meta de la Agenda 2030 asociada, llegando a establecer la siguiente matriz:

Lideramos la lucha contra el cambio climático en la gestión del agua
Alcanzar una reducción del g0\% de las emisiones de CO2 derivadas de nuestro consumo eléctrico




\section{INFORMAR, INTEGRAR E IMPLEMENTAR EL CAMBIO}

El compromiso ante los ODS de las organizaciones sólo existe cuando se hace público y es conocido por todos los grupos de relación implicados. De este modo, la organización expresa y comunica de forma eficaz, sencilla y práctica, sus aspiraciones en materia de desarrollo sostenible, inspirando y comprometiendo a empleados, inversores, clientes y otros grupos de relación, con el riesgo que conlleva. Es necesario comunicar, de forma regular y transparente, acerca de los esfuerzos realizados, los progresos alcanzados y los desafíos que se afrontan.

En SUEZ se lleva a cabo un seguimiento anual, a través de su informe de desarrollo sostenible, del progreso de los indicadores asociados a cada compromiso y a cada ODS. Además, a través del apartado "Palabras del Presidente" del informe, se refuerza el compromiso y posicionamiento de la organización en materia de los ODS. Uno de los grandes retos es disponer de datos actualizados en todo momento para poder rendir cuentas en continuo del estado de cada uno de los compromisos estratégicos establecidos en el plan de desarrollo sostenible.

Para integrar los objetivos de sostenibilidad en el negocio, la implicación de la dirección es esencial. Por ello, en SUEZ han sido ejecutados procesos formativos específicos para todos los gerentes de las empresas del grupo con el fin de que integren los ODS en los Consejos de Administración.

Aunque la dirección y los equipos de sostenibilidad dedicados pueden desempeñar un papel importante en el logro de los objetivos de sostenibilidad de la compañía, el apoyo y la participación de las funciones corporativas trasversales como la innovación, el desarrollo de negocios, compras, operaciones y recursos humanos, son clave para la incorporación de la sostenibilidad en la estrategia de los negocios, la cultura y las operaciones. En SUEZ cada área es responsable del diseño, ejecución y seguimiento de uno o más de los 10 compromisos del REwater Global Plan relacionados con su ámbito.

Como conclusión, analizar y priorizar los ODS es necesario antes de desarrollar un plan de acción, un posicionamiento y una estrategia en desarrollo sostenible que debe ser impulsada por la dirección y por todas las áreas de la organización. Sólo de este modo, los ODS serán una palanca de cambio y transformación en las organizaciones para lograr el desarrollo sostenible. 